\title{
COVID19 Associated Thrombotic Angiopathy Improved After Plasma Exchange
}

Natalie Elkayam ${ }^{1}$ Gagan Raju , Martin Bluth², Yiwu Huang ${ }^{2}$, Jay Lipshitz ${ }^{2}$, and Stephen Peeke $^{2}$

${ }^{1}$ Maimonides Cancer Center

${ }^{2}$ Maimonides Medical Center

March 8, 2021

\begin{abstract}
Woman admitted for COVID-19 respiratory failure requiring intubation, renal failure and rising bilirubin, requiring CVVHD. Due to dropping hemoglobin and platelets, TTP was suspected and empiric plasma exchange initiated. Platelets normalized; she improved; ADAMTS13 level resulted 50.7\%, indicating possible benefit of plasma exchange for COVID19 thrombotic microangiopathy despite normal ADAMTS13.
\end{abstract}

\section{COVID19 Associated Thrombotic Angiopathy Improved After Plasma Exchange}

Natalie Elkayam, M.D. ${ }^{1}$; Gagan Raju, M.D. ${ }^{1}$; Bluth, Martin, M.D. Ph.D. ${ }^{2}$; Huang, Yiwu, M.D. ${ }^{1}$; Lipshitz, Jay, M.D. ${ }^{1}$; Peeke, Stephen, M.D. ${ }^{1}$

${ }^{1}$ Department of Hematology and Oncology, Maimonides Medical Center, Brooklyn, NY, USA

${ }^{2}$ Department of Pathology, Maimonides Medical Center, Brooklyn, NY, USA

Corresponding author: Gagan Raju, MD (gagan.raju@gmail.com)

Case presentation:

A 44-year-old Chinese woman with no known medical history presented to the emergency department for evaluation of shortness of breath. The patient reported fevers, chills and a dry cough which had progressively worsened during the preceding week. Her husband was also sick at home with similar symptoms. Upon arrival to the emergency room, she was afebrile (36.4) with a blood pressure of $132 / 87 \mathrm{mmHg}$, tachycardic (122 beats/minute) and tachypneic (25 breaths/minute). Her oxygen saturation was $80 \%$ on $6 \mathrm{~L}$ of oxygen via nasal cannula requiring escalation to a non-rebreather mask on which her oxygen saturation declined to $75 \%$. Due to progressive dyspnea and hypoxia refractory to oxygen supplementation, she was endotracheally intubated and mechanically ventilated on account of acute respiratory failure. Initial laboratory studies demonstrated creatinine of $1.0 \mathrm{mg} / \mathrm{dL}$, lactate dehydrogenase (LDH) was 2334IU/L and liver function tests (LFT) were aspartate transaminase (AST)/alanine transaminase (ALT) 79/63 IU/L with bilirubin levels within normal limits. Coronavirus-2019 (COVID19) PCR was reactive. C-reactive protein (CRP) was $38.6 \mathrm{mg} / \mathrm{dL}$ and ferritin was found to be $297.9 \mathrm{ng} / \mathrm{ml}$. Her complete blood count (CBC) on presentation demonstrated the following: white blood cell (WBC) 13.4 cells/L, hemoglobin $(\mathrm{Hgb})$ of $11.7 \mathrm{~g} / \mathrm{dL}$ and platelet count of $243 \mathrm{k} / \mathrm{uL}$. Coagulation profile including international normalized ratio (INR), activated partial thromboplastin time (aPTT) and fibrinogen were all in normal range. Patient was admitted to the medical intensive care unit for management of septic shock and acute respiratory failure secondary to 
acute COVID19 infection. She underwent CT angiography of the chest, which demonstrated no pulmonary embolism and bilateral, predominantly ground glass opacities consistent with COVID19 infection changes.

The patient received dexamethasone therapy as indicated in the standard of care for management of severe COVID19 infection. Within one day of her hospitalization, her laboratory findings demonstrated worsening renal function (blood urea nitrogen $(\mathrm{BUN}) /$ creatinine $45 / 2.2 \mathrm{mg} / \mathrm{dL}$ ), and worsening total bilirubin up to $4.0 \mathrm{~mL} / \mathrm{dL}$ within 3 days of her admission. The patient underwent continuous venovenous hemodiafiltration (CVVHD) for acute renal failure. Her troponin trended up to $1.41 \mathrm{ng} / \mathrm{mL}$, which was attributed to demand ischemia due to underlying infectious process. Her WBC count remained stable throughout her admission. Her hemoglobin decreased to $6.8 \mathrm{~g} / \mathrm{dL}$ and she received PRBC transfusion. Her platelet count within one day of admission dropped from 243 to $65 \mathrm{k} / \mu \mathrm{L}$ and further decreased to $23 \mathrm{k} / \mu \mathrm{L}$. Poly Coombs test was negative. Heparin induced thrombocytopenia (HIT) antibody was negative.

Due to dropping hemoglobin and platelet values, hematology evaluation was obtained for assessment of possible thrombotic thrombocytopenic purpura (TTP). Peripheral smear demonstrated normochromic red blood cells (RBC) with some polychromasia, numerous nucleated RBCs, occasional basophilic stippling with many schistocytes identified (approximately 5-6/high power field). No platelet clumps were identified though some large platelets were observed and manual platelet count estimated around 30-50k. Polymorphonuclear cells with toxic granules, many band cells and some large activated lymphocytes were identified. ADAMTS13 testing was sent.

On account of high clinical suspicion for thrombotic angiopathy, a decision was made to commence empiric plasma exchange in advance of receipt of ADAMTS13 level. The patient underwent plasma exchange with one blood volume of fresh frozen plasma (FFP) for five consecutive days.

After initiation of plasma exchange, the patient's laboratory values demonstrated significant improvement: LDH level improved from 3802 to 945 within one day of plasma exchange initiation. Total bilirubin levels improved from 4.0 to 2.9 within one day of plasma exchange. After the initial plasma volume exchange, her platelet count increased from $18 \mathrm{k}$ to $68 \mathrm{k}$ and continued to increase and eventually normalize thereafter, concurrent with daily plasma exchange. The patient also showed significant clinical improvement with declining vasopressor requirement after the second day of plasma exchange and subsequently required antihypertensives due to elevated blood pressure on the third day of plasma exchange. Peripheral smear on the fourth day of plasma exchange demonstrated a significant decrease in schistocytes per high power field. The presumption was that the rapid clinical improvement concurrent with plasma exchange was consistent with the diagnosis of TTP. However after five consecutive plasma exchanges, the ADAMTS13 level (drawn before initiation of plasma exchange) resulted at 50.7\%, subsequently plasma exchange was discontinued. The patient no longer required renal replacement therapy.

\section{Hosted file}

image1. emf available at https://authorea.com/users/400277/articles/512609-covid19-associatedthrombotic-angiopathy-improved-after-plasma-exchange

Figure 1. Peripheral smear with numerous schistocytes seen.

\section{Differential Diagnosis}

The patient initially presented with normal platelet count and with normal mentation. The rapid onset of thrombocytopenia and renal failure in the context of severe COVID19 infection raised the concern for possible thrombocytopenia induced by consumptive process due to her underlying infection. In addition, COVID19-induced thrombotic angiopathy without TTP was also considered on account of the patient's coagulopathic and inflammatory changes. The presence of a significant number of schistocytes on peripheral smear raised the concern for thrombotic thrombocytopenic purpura (TTP), despite initial presentation with normal platelet count and concurrent COVID19 infection. Due to a high clinical and laboratory suspicion for TTP, plasma exchange with FFP was initiated with pending ADAMTS13 results. The patient experienced a significant improvement in her clinical status, biochemically, by laboratory values and with significant 
decrease in schistocyte per high power field. The patient's coagulation profile was not consistent with disseminated intravascular coagulation (DIC). On account of normal ADAMTS13 levels, yet clear laboratory and pathologic evidence of a thrombotic microangiopathy, our patient was diagnosed with COVID19-induced thrombotic angiopathy, which responded briskly to plasma exchange intervention.

Discussion

Patients with COVID19 infection have been found to exhibit a wide array of symptoms and complications, as published in the literature in 2020. There is a known association with COVID19 infection and a unique coagulopathy. It is postulated that this coagulopathy may be related to endothelial activation and microvascular thrombosis/hemolysis. There is evidence that viruses play an important role as a trigger in the pathogenesis of thrombotic angiopathies. The mechanism of this remains unclear; it has been suggested that direct endothelial injury by cytokine storm and immune complex mediated events along with ADAMTS13 inhibitors are implicated as underlying triggers of viral activated thrombotic microangiopathy [1].

A study evaluating secondary thrombotic microangiopathy in COVID19-infected patients found that patients with low ADAMTS13 levels, elevated LDH, presence of schistocytes and elevated von Willebrand factor levels were more likely to indicate severe infection and high likelihood of death [2].

There are case reports of secondary TTP due to COVID19 infection. There is also a report by Albiol et al. of a 57-year-old woman diagnosed with acquired autoimmune TTP following the diagnosis of Covid-19 [3]. Another case is reported by Hindilerden et al. of a case of TTP which was diagnosed following COVID19 infection [4].

Martinelli et. al carried out a retrospective study on 50 admitted patients with COVID19 infection in which they evaluated various laboratory values. They found that about $2-4 \%$ of patients had documentation of schistocytes on peripheral smears. They also found a mild decrease in ADAMTS13 level in most of the subjects (47\% with 95\% CI 40-55). This was the first study suggesting ADAMTS13 impairment in COVID19 infection [5].

Our patient had a COVID-19-associated thrombotic microangiopathy that exhibited a dramatic response to plasma exchange. The current report adds to a growing body of literature reporting improvement with plasma exchange in severely ill patients with COVID19 infection [6][7]. It is conceivable that plasma exchange accounts for cytokine removal of inflammatory mediators in the plasma and reinstitution of immune homeostasis. While employing plasma exchange as a therapeutic modality for COVID-19 is sure to pose significant challenges, the emerging evidence warrants further scientific consideration. Plasma exchange used on a limited scale, particularly for patients with COVID-19 microangiopathy, may represent a useful treatment for a particularly devastating manifestation of COVID-19.

References:

1. Lopes da Silva R. Viral-associated thrombotic microangiopathies. Hematol. Oncol. Stem Cell Ther. 2011;4(2):51-59. doi: 10.5144/1658-3876.2011.51.

2. Sweeney JM, Barouqa M, Krause GJ, Gonzalez-Lugo JD, Rahman S, Gil MR. Evidence for secondary thrombotic microangiopathy in COVID-19. Preprint. medRxiv . 2020;2020.10.20.20215608. Published 2020 Oct 23. doi:10.1101/2020.10.20.20215608

3. Albiol N., Awol R., Martino R. Autoimmune thrombotic thrombocytopenic purpura (TTP) associated with COVID-19. Ann. Hematol. 2020 May 28:1-2. doi: 10.1007/s00277-020-04097-0.

4. Hindilerden, Fehmi et al. "Covid-19 associated autoimmune thrombotic thrombocytopenic purpura: Report of a case." Thrombosis research vol. 195 (2020): 136-138. doi:10.1016/j.thromres.2020.07.005

5. Martinelli, N., Montagnana, M., Pizzolo, F., Friso, S., Salvagno, G., Forni, G., Luca et al. (2020). A relative ADAMTS13 deficiency supports the presence of a secondary microangiopathy in COVID 19. Thrombosis Research. 193. . 2020 Sep; 193: 170-172

6. Shi H., Zhou C., He P. Successful treatment of plasma exchange followed by intravenous immunoglobin in a critically ill patient with 2019 novel coronavirus infection. Int J Antimicrob Agents. 2020:105974. 
doi: $10.1016 /$ j.ijantimicag.2020.105974.

7. Zhang L., Zhai H., Ma S., Chen J., Gao Y. Efficacy of therapeutic plasma exchange in severe COVID-19 patients. Br J Haematol. 2020 doi: 10.1111/bjh.16890

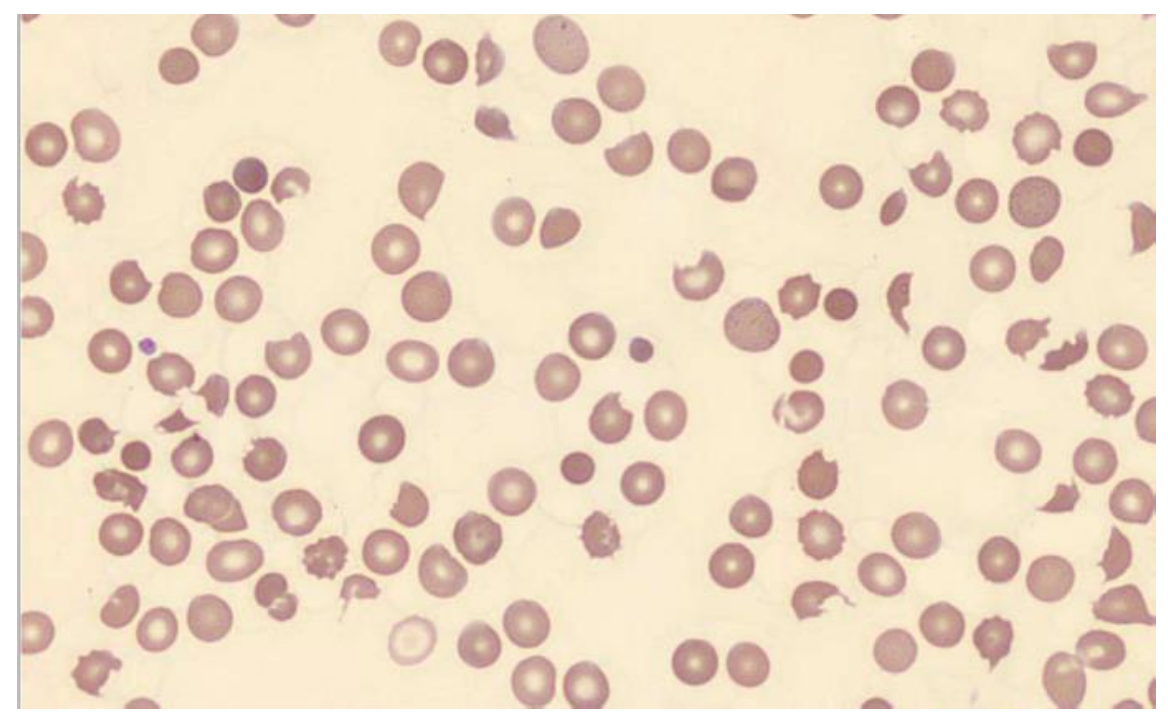

